\title{
Beyond Expert Opinion: A Comparison of Antibiotic Regimens for Infectious Urinary Tract Pathology in Pregnancy
}

\author{
Madison K. Krischak, BS ${ }^{1}$ Heather A. Rosett, MD ${ }^{1}$ Sarika Sachdeva, BS ${ }^{1}$ Kristin E. Weaver, BS $^{2}$ \\ Robert Phillips Heine, MD ${ }^{3}$ Anna E. Denoble, MD, MSCR ${ }^{2}$ Sarah K. Dotters-Katz, MD, MMHPE ${ }^{2}$
}

1 School of Medicine, Duke University, Durham, North Carolina

2 Department of Obstetrics and Gynecology, Duke University, Durham, North Carolina

${ }^{3}$ Department of Obstetrics and Gynecology, Wake Forest Baptist Medical Center, Winston-Salem, North Carolina

\begin{abstract}
Address for correspondence Madison K. Krischak, BS, Duke University School of Medicine, 2608 Erwin Road, Suite 210, Durham, NC 27705 (e-mail: madison.krischak@duke.edu).
\end{abstract}

Am J Perinatol Rep 2020;10:e352-e356.

\begin{abstract}
Keywords

- asymptomatic bacteriuria

- acute cystitis

- nitrofurantoin

- pyelonephritis

- trimethoprimsulfamethoxazole

- uropathogen

- urinary tract infections

Objective Outside pregnancy, nitrofurantoin, ciprofloxacin and sulfamethoxazoletrimethoprim (SMZ-TMP) are first-line therapy (FLT) for lower urinary tract infections (LUTIs). Optimal antibiotics for LUTI have been extrapolated based on expert opinion. Progression to pyelonephritis and adverse obstetric outcomes were compared between women who received FLT and those given alternative antibiotics.

Methods This study includes a retrospective cohort of women with LUTI, including asymptomatic bacteriuria and acute cystitis at single health care system from July 2013 to May 2019. Women receiving FLT, defined as nitrofurantoin or SMZ-TMP, were compared with those receiving nonfirst-line therapy (nFLT). Primary outcome was progression to pyelonephritis. Secondary outcomes included pyelonephritis-related anemia, sepsis, length of stay, preterm birth (PTB), and low birth weight (LBW). Logistic regression was used to calculate odds of outcomes.

Results Of 476 women, $336(70.6 \%)$ received FLT and $140(29.4 \%)$ received nFLT. Women receiving FLT were more likely having BMI $\geq 40(p=0.04)$. Progression to pyelonephritis did not differ ( 5.8 vs. $8.2 \% ; p=0.44$ ), nor did other pyelonephritisrelated outcomes. After controlling for confounders, no difference in odds of progression to pyelonephritis was seen (adjusted odds ratio [aOR] 1.02, 95\% confidence interval $[\mathrm{Cl}] 0.42,2.49$ ). FLT was not associated with PTB or LBW (aOR 0.60, 95\% $\mathrm{Cl} 0.29$, 1.26) after controlling for confounders.

Conclusion Receipt of antibiotics other than nitrofurantoin or SMZ-TMP for LUTI in pregnancy was not associated with increased risk of progression to pyelonephritis, PTB, or LBW.
\end{abstract}

Lower urinary tract infections (LUTIs) are one of the most common medical complications of pregnancy, occurring in $8 \%$ of U.S. women. ${ }^{1}$ LUTIs include both asymptomatic bacteriuria (ASB) or acute cystitis (AC). Left untreated, ASB develops into symptomatic $A C$ in $30 \%$ of patients and may progress to pyelonephritis in up to $50 \%$ of those patients. ${ }^{2}$ It is estimated that 0.5 to $2 \%$ of all pregnant women are hospitalized for treatment of pyelonephritis. ${ }^{3,4}$ Pyelonephritis is associated with increased risk of anemia, sepsis, acute pulmonary insufficiency, acute renal dysfunction, and received

June 13, 2020

accepted after revision

July 20,2020
DOI https://doi.org/

$10.1055 / \mathrm{s}-0040-1718384$ ISSN 2157-6998.
Copyright $\odot 2020$ by Thieme Medical

Publishers, Inc., 333 Seventh Avenue, New York, NY 10001, USA Tel: +1(212) 760-0888
License terms

(ㅇ)(1) $\Theta \circledast$ 
spontaneous preterm birth (PTB). ${ }^{3}$ Given the increased maternal morbidity of untreated LUTI, the United States Preventive Services Task Force recommends routine screening for and treatment of ASB and AC during pregnancy. ${ }^{5}$

Treatment of ASB and AC in pregnancy must cover the most common uropathogens, considering the altered pharmacokinetics, and must take into account teratogenicity and fetal toxicity of some antibiotics during pregnancy. The most common urinary tract pathogen is Escherichia coli, accounting for 80 to $90 \%$ of UTIs in pregnancy. ${ }^{1}$ Other common UTI pathogens include Group-B Streptococcus, Staphylococcus saprophyticus, Klebsiella pneumoniae, and Proteus mirabilis. Many antibiotics offer sufficient coverage of these microorganisms; however, antibiotic toxicity in pregnancy restricts treatment options. Antimicrobial agents currently approved for use in pregnancy include nitrofurantoin, $\beta$ lactams, cephalosporins, and fosfomycin trometol. ${ }^{6}$ However, current American College of Obstetricians and Gynecologists recommendations identify nitrofurantoin and sulfonamides, primarily trimethoprim-sulfamethoxazole (SMZ-TMP), as first-line therapies for LUTI in pregnancy. ${ }^{7}$

Despite these recommendations, a recent CDC study that used MarketScan Commercial Database to find pregnancies with UTIs and filled antibiotic prescriptions, and included 450,000 pregnancies of which nearly 40,000 met inclusion criteria, found that less than half of LUTI were treated with nitrofurantoin or SMZ-TMP. ${ }^{8}$ These data suggest nonfirst-line antibiotics are commonly used to treat LUTI in pregnancy. However, the efficacy in preventing progression to pyelonephritis of nonfirst-line antibiotics for UTI in pregnancy is unknown. The purpose of this study is to compare the rates of progression to pyelonephritis and obstetric outcomes among women treated with first-line antibiotic therapies, specifically nitrofurantoin or SMZ-TMP, with those treated with nonfirst-line therapies.

\section{Materials and Methods}

This was a retrospective cohort study of all pregnant women presenting at a single health system with LUTI, including ASB or AC, during pregnancy. Women delivering at a Duke University-affiliated hospital from July 1, 2013 to May 1, 2019 were included. Eligible patients were identified using International Classification of Diseases, 9th (ICD-9) and 10th (ICD-10) Revision codes for ASB, acute cystitis, and pyelonephritis.

Women were included if they were diagnosed with LUTI during pregnancy and delivered during the study period in the health care system. Women with missing culture data, antibiotic data, or delivery information were excluded. Women with documentation of medication nonadherence per provider records were also excluded. ASB and AC were defined as urine culture positive for a single organism $\geq 10^{5}$ $\mathrm{cfu} / \mathrm{mL}$ without symptoms and $\geq 10^{2} \mathrm{cfu} / \mathrm{mL}$ with symptoms, respectively. ${ }^{9}$ Pyelonephritis was defined by clinical diagnosis documented by the provider during admission.

The electronic medical record was reviewed, and demographic variables, antepartum and pregnancy complications, delivery, and postpartum data were abstracted by trained chart abstractors. The exposure of interest was receipt of a "first-line" antibiotic for the treatment of LUTI. First-line antibiotic therapy was defined as nitrofurantoin or SMZTMP. ${ }^{10}$ Nonfirst-line therapy included all other antibiotics. Antibiotic choice was at the discretion of the provider.

The primary outcome was progression to pyelonephritis. Secondary outcomes included length of antibiotic course, pyelonephritis-related anemia (defined as the hematocrit nadir during the admission for pyelonephritis), sepsis, pyelonephritis length of stay, preterm delivery, and low birth weight. Preterm delivery was defined as delivery prior to 37 weeks. Short course of antibiotics was defined as less than 7 days. Maternal anemia was defined as a hematocrit nadir less than $32 \% .{ }^{11}$ Women who received first-line antibiotic therapy (FLT) were compared with those who received nonfirst-line therapies (nFLTs).

Baseline demographics were analyzed with Student's t-test, Wilcoxon rank sum tests, Chi-square tests, or Fisher's exact tests as appropriate. Multivariate logistic regression was used to determine significant predictors of the primary outcome. Statistical significance was defined as $p<0.05$. Statistical analysis was performed using STATA software version 14.0. This study was approved by the Duke University IRB.

\section{Results}

Of 722 women with LUTI, 476 women met inclusion criteria with culture-confirmed LUTI and documentation of antibiotic therapy completion (-Fig. 1). Of these, 336 (70.6\%)

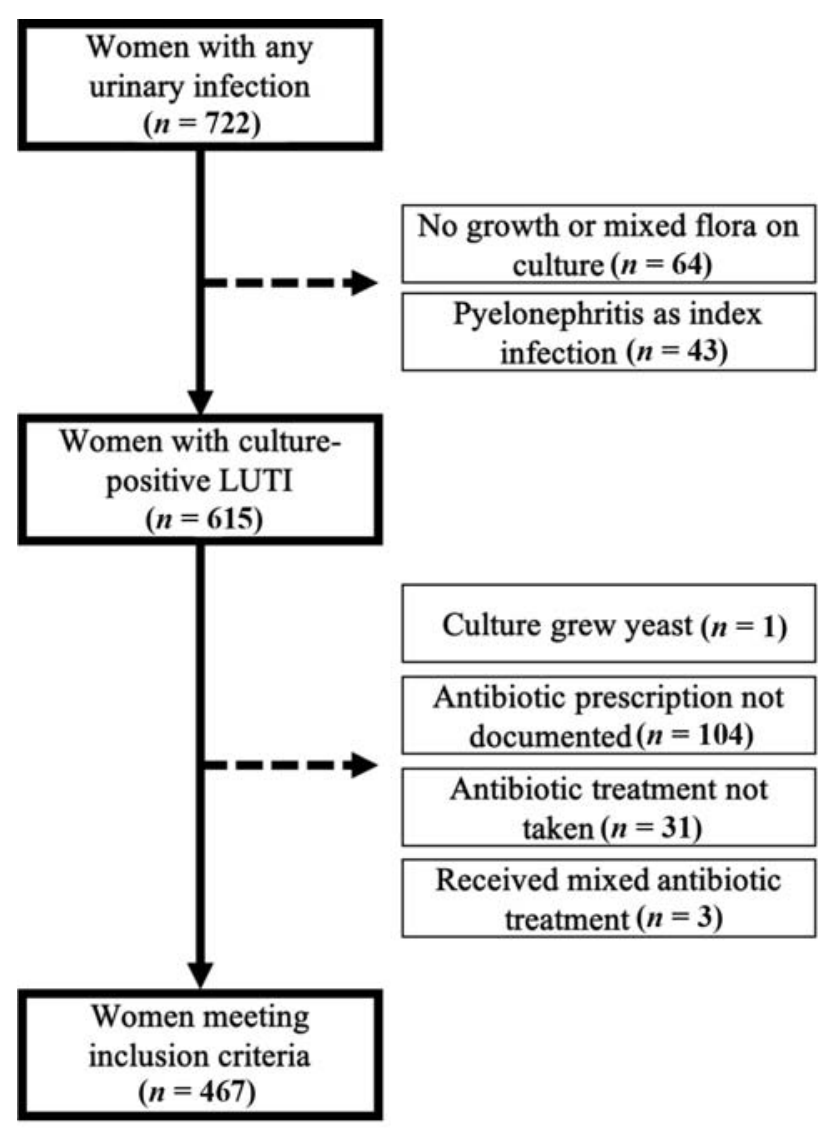

Fig. 1 Study population. 
e354 Antibiotic Treatment of UTI and Progression to Pyelonephritis Krischak et al.

Table 1 Antibiotics received among women with UTI and ASB

\begin{tabular}{|l|l|}
\hline Antibiotic & $N(\%)$ \\
\hline First-line therapy & $335(71)$ \\
\hline Nitrofurantoin & $322(68)$ \\
\hline SMZ-TMP & $13(3)$ \\
\hline Nonfirst-line therapy & $140(29)$ \\
\hline Ampicillin & $60(13)$ \\
\hline Cephalexin & $49(10)$ \\
\hline Other & $31(6)$ \\
\hline
\end{tabular}

Abbreviations: ASB, asymptomatic bacteriuria; SMZ-TMP, trimethoprimsulfamethoxazole; UTI, urinary tract infection.

received nitrofurantoin or SMZ-TMP and 140 (29.4\%) received nFLT. The most common nFLT used was ampicillin (13\%), followed by cephalexin (10\%). Sixty-eight percent of women received nitrofurantoin; this was the most common treatment and the most common FLT ( - Table $\mathbf{1}$ ).

Women who received FLT were more likely to have a BMI $>40(p=0.04)$ than those who received nFLT ( - Table 2 ). Other baseline demographic data did not differ ( - Table 2 ). Women who were treated with nFLT were more likely to receive a short course of their prescribed antibiotic (-Table 2).

E. coli was the single most common pathogen, occurring in $41.8 \%(n=199)$ of all positive cultures. Among women receiving FLT, $48.5 \%$ had a culture positive for E. coli, com- pared with only $25.7 \%$ of women who received nFLT, $(p<0.01)$. Group B Strep LUTI were also common, occurring in $31.7 \%(n=151)$ of all cultures. Women with GBS LUTI were less likely to receive FLT, (24.1\% FLT vs. $50.0 \%$ nFLT, $p<0.01)$.

Among the 476 women included in the analysis, 35 (7.3\%) developed pyelonephritis. Progression to pyelonephritis did not differ by receipt of FLT, ( 8.2 vs. $5.8 \%, p=0.44$ ). After controlling for history of UTI, culture result, treatment duration $<7$ days, and BMI $\geq 40$, no difference in odds of progression to pyelonephritis was seen between women who received FLT and those that did not (adjusted odds ratio 1.02 , 95\% confidence interval $0.42,2.49$ ). Pyelonephritisassociated morbidities were uncommon. However, of those women who progressed to pyelonephritis in the nFLT and FLT groups, a trend toward increased rates of sepsis was seen in those who received nFLT ( $2 / 8$ vs. $0, p=0.05)$. Regarding obstetric outcomes, receipt of FLT was also not associated with earlier gestational age at delivery, PTB, or LBW (-Table 3), even after controlling for confounders (data not shown).

\section{Comment}

Among women with LUTI, receipt of nonfirst-line antibiotic therapy was not associated with higher odds of progression to pyelonephritis. Pyelonephritis-related sepsis was uncommon in both groups; however, it occurred in $25 \%$ of women in the nFLT compared with no women in the FLT group. No other pyelonephritis-related morbidities differed by antibiotic receipt, nor did obstetric outcomes.

Table 2 Maternal demographics among women with ASB and UTI by antibiotic treatment regimen ${ }^{\text {a }}$

\begin{tabular}{|l|l|l|l|}
\hline & $\begin{array}{l}\text { Non-1st line Tx } \\
\mathbf{n}=\mathbf{1 4 0}(\%)\end{array}$ & $\begin{array}{l}\text { 1st line Tx } \\
\boldsymbol{n}=335(\%)\end{array}$ & $p$-Value \\
\hline Median age, years (IQR) & $27(22,32)$ & $28(23,33)$ & 0.34 \\
\hline African American Race & $31(22.1)$ & $83(24.7)$ & 0.64 \\
\hline Private insurance & $43(31.4)$ & $116(35.1)$ & 0.15 \\
\hline Limited prenatal care & $7(5.1)$ & $14(4.2)$ & 0.81 \\
\hline Chronic hypertension & $16(11.5)$ & $23(6.9)$ & 0.10 \\
\hline Diabetes mellitus & $9(6.5)$ & $17(5.1)$ & 0.52 \\
\hline Tobacco use & $14(10.1)$ & $36(10.8)$ & $>0.99$ \\
\hline BMI $\geq 40$ & $29(20.7)$ & $43(12.8)$ & 0.04 \\
\hline Median baseline hematocrit (IQR) & $36.6(35.0,39.9)$ & $37.0(34.6,39.0)$ & 0.49 \\
\hline Depression & $15(10.7)$ & $68(20.2)$ & 0.01 \\
\hline Multiparous & $81(57.9)$ & $213(63.4)$ & 0.30 \\
\hline Prior preterm birth & $24(17.1)$ & $52(15.5)$ & 0.68 \\
\hline History of pyelonephritis & $4(2.9)$ & $14(4.2)$ & 0.61 \\
\hline History of UTI & $27(19.3)$ & $90(26.9)$ & 0.10 \\
\hline Median GA of first infection, wk, (IQR) & $11.0(8.7,21.3)$ & $11.6(9.3,18.4)$ & 0.54 \\
\hline Short therapy (<7 d) & $16(11.4)$ & $12(3.6)$ & $<0.01$ \\
\hline
\end{tabular}

Abbreviations: ASB, asymptomatic bacteriuria; BMI, body mass index; GA, gestational age; IQR, interquartile range; Tx, therapy; UTI, urinary tract infection.

${ }^{\mathrm{a}}$ Data presented as $n(\%)$, unless otherwise stated. 
Table 3 Maternal and obstetric outcomes among women with ASB and UTI by antibiotic treatment regimen ${ }^{a}$

\begin{tabular}{|l|l|l|l|}
\hline & $\begin{array}{l}\text { Non-1st line Tx } \\
\boldsymbol{n}=\mathbf{1 4 0}(\%)\end{array}$ & $\begin{array}{l}\text { 1st line Tx } \\
\boldsymbol{n}=\mathbf{3 3 6}(\%)\end{array}$ & $p$-Value \\
\hline Progression to pyelonephritis & $8(5.8)$ & $27(8.2)$ & 0.44 \\
\hline Median pyelonephritis LOS, days, (IQR) & $3.5(2,5)$ & $4(2,5)$ & 0.97 \\
\hline Median hematocrit nadir (IQR) & $26.7(24.4,29.9)$ & $29.9(27.0,31.8)$ & 0.12 \\
\hline Anemia & $7 / 9(77.8)$ & $21 / 27(77.8)$ & 0.99 \\
\hline Blood transfusion & $1 / 8(12.5)$ & 0 & 0.23 \\
\hline Sepsis & $2 / 8(25.0)$ & 0 & 0.05 \\
\hline Median GA delivery, weeks, (IQR) & $39.3(37.7,40.0)$ & $39.1(37.9,40.0)$ & 0.94 \\
\hline Preterm birth & $13(9.3)$ & $42(12.5)$ & 0.35 \\
\hline Low birth weight & $15(10.7)$ & $23(6.9)$ & 0.19 \\
\hline
\end{tabular}

Abbreviations: ASB, asymptomatic bacteriuria; GA, gestational age; IQR, interquartile range; LOS, length of stay; Tx, therapy; UTI, urinary tract infection.

${ }^{\mathrm{a}}$ Data presented as $n(\%)$, unless otherwise stated.

Treatment of LUTI in pregnancy is restricted due to antibiotic safety and toxicity. Outside of pregnancy, where antibiotic selection is not limited by pregnancy-specific safety concerns, the recommended therapies for UTI in women include fosfomycin, nitrofurantoin, and SMZTMP. ${ }^{12}$ Unlike $\beta$-lactams, these first-line agents in nonpregnant women have less microbial resistance and achieve higher rates of complete clearance of gram-negative microorganisms from the urine, decreasing LUTI recurrence rates. $^{13}$ In pregnancy, guidelines identify nitrofurantoin and SMZ-TMP and first-line treatment of LUTI in pregnancy based on efficacy and teratogenicity. ${ }^{10} \mathrm{~A}$ large recent observational study of more than 450,000 pregnancies found that only $42.3 \%$ of first trimester LUTI are treated with FLTs of nitrofurantoin or SMZ-TMP. ${ }^{8}$ In the current study, $70.6 \%$ of women were treated with first-line therapies suggesting that national rates of first-line therapy may vary by institution and provider preferences. A 2011 Cochrane review of 1,125 women treated for AC in pregnancy found no significant differences between antibiotic treatments with regards to infection cure rate, but did not assess progression to pyelonephritis. ${ }^{14}$ This study also noted that it was not possible to draw conclusion on the best class of antibiotic nor the duration, and went on to say that "Future studies should evaluate the more promising classes of antibiotics, such as nitrofurantoin, trimethoprim-sulfamethoxazole..."14 Thus, more data are needed. However, the findings of our study appear consistent with these results and are more current; given that existing data suggest that cure rates are similar, progression rates also likely do not differ.

Use of nFLT for treatment of LUTI in pregnancy does not appear to carry increased risk of progression to pyelonephritis or worse obstetric outcomes. These findings suggest that antibiotic choice for LUTI in pregnancy may not need to be limited to the recommended first-line therapies. Thus, the choice of antibiotics for treatment of LUTI can be based on other factors including provider or patient preference, local resistance patterns, and side effect profiles. Further larger studies are needed to assess rates of progression to pyelone- phritis among individual antibiotics within the FLT and nFLT groups. Additionally, prospective randomized control trials could provide more data on ideal FLT in pregnancy.

This study has multiple strengths. First, the study was conducted over 6 years at a large health care system allowing for a representative sample of antibiotic therapies used for LUTI in pregnancy. Additionally, the study population was diverse across metrics of age, race, ethnicity, and socioeconomic status making it generalizable to the broader population. We also recognize several limitations of the study. In this study, antibiotic therapies were simplified into only two groups: FLT and nFLT. Individual antibiotics within the respective groups may not share the same outcomes as others in the group. Given the limited sample size of this cohort, we were only able to analyze between general therapy groups. The sample size thus limits the ability to differentiate among therapies and provide detailed outcomes for each individual therapy. We also recognize that among gram-negative infections in this cohort, there is some degree of antibiotic resistance; however, in the adjusted analysis, we controlled for infectious pathogen to mitigate this to some extent. Another limitation concerns the rate that a test of cure was performed within 2 weeks of treatment, only $12 \%$ of the time, which limits our ability to comment on effectivity of treatment. However, a larger analysis could be completed to evaluate outcomes of each antibiotic independently. We are also limited by a small sample size. With the current sample size, a significance level of 0.05 , and assuming an increase from 5 to $10 \%$ incidence of pyelonephritis as a clinically important difference to detect, the power of this study to exclude a type II error is $45 \%$. Achieving $80 \%$ power and assuming the same clinically important difference to detect would require 474 subjects per group. Thus, there is a chance of type 2 error. Additionally, very few women, only $12 \%$, had a test of cure within 2 weeks of completion of therapy, limiting our ability to comment on bacterial clearance. We are also unable to explain antibiotic prescribing that varied from our standing protocols which recommend nitrofurantoin, cephalexin, or SMZ-TMP, as this occurred at the discretion of the provider (of which there are 
over 100 involved in the care of pregnant women at our institution). Lastly, the rate of progression to pyelonephritis in this study was found to be $7.3 \%$ whereas prior large analyses demonstrate the rate of $1.4 \%{ }^{15}$ This could suggest that our population of patients was at higher risk of pyelonephritis than seen nationally regardless of the treatments used, making the results less generalizable.

LUTIs in pregnancy treated with nonfirst-line antibiotics do not appear to progress to pyelonephritis at higher rates than those treated with nitrofurantoin or SMZ-TMP. Additionally, no difference was seen in obstetric outcomes in women treated with first-line antibiotics versus nonfirst-line antibiotics. Future studies are required to determine whether individual antibiotics used in pregnancy, regardless of first-line or nonfirst-line status, result in higher rates of adverse obstetric outcomes or rates of progression to pyelonephritis.

\section{Highlights}

- As per expert opinion, nitrofurantoin and sulfamethoxazole-trimethoprim (SMZ-TMP) are first line for lower urinary tract infections (LUTI) in pregnancy.

- Nonfirst-line drugs do not increase the rate of LUTI progression to pyelonephritis.

- Pyelonephritis and obstetric outcomes are similar regardless of antibiotic choice.

\section{Financial Support}

None to disclose.

\section{Meeting Presentation Disclosure}

This work was presented as a poster presentation at the Society for Maternal-Fetal Medicine's 40th Annual Pregnancy meeting in February 2019 in Dallas, Texas.

\section{Condensation}

Receipt of antibiotics other than nitrofurantoin or trimethoprim-sulfamethoxazole for LUTI in pregnancy is not associated with increased risk of progression to pyelonephritis.
Disclosure Statement

The authors report no conflict of interest.

\section{References}

1 Delzell JE Jr, Lefevre ML. Urinary tract infections during pregnancy. Am Fam Physician 2000;61(03):713-721

2 Kass EH. Pregnancy, pyelonephritis and prematurity. Clin Obstet Gynecol 1970;13(02):239-254

3 Wing DA, Fassett MJ, Getahun D. Acute pyelonephritis in pregnancy: an 18-year retrospective analysis. Am J Obstet Gynecol 2014;210(03):219.e1-219.e6

4 Bacak SJ, Callaghan WM, Dietz PM, Crouse C. Pregnancy-associated hospitalizations in the United States, 1999-2000. Am J Obstet Gynecol 2005;192(02):592-597

5 Force UPST U.S. Preventive Services Task Force. Screening for asymptomatic bacteriuria in adults: U.S. Preventive Services Task Force reaffirmation recommendation statement. Ann Intern Med 2008;149(01):43-47

6 Smaill FM, Vazquez JC. Antibiotics for asymptomatic bacteriuria in pregnancy. Cochrane Database Syst Rev 2015;(08):CD000490

7 Gynecologists ACoOa. Sulfonamides, nitrofurantoin, and risk of birth defects. Committee Opinion No. 717. Obstet Gynecol 2017; 130:e150-e152

8 Ailes EC, Summers AD, Tran EL, et al. Antibiotics dispensed to privately insured pregnant women with urinary tract infectionsUnited States, 2014. MMWR Morb Mortal Wkly Rep 2018;67(01): $18-22$

9 Stamm WE. Recent developments in the diagnosis and treatment of urinary tract infections. West J Med 1982;137(03):213-220

10 Gynecologists ACoOa. American College of Obstetricians and Gynecologists Committee on Obstetric Practice. ACOG Committee Opinion No. 494: sulfonamides, nitrofurantoin, and risk of birth defects. Obstet Gynecol 2011;117:1484-1485

11 American College of Obstetricians and Gynecologists. ACOG Practice Bulletin No. 95: anemia in pregnancy. Obstet Gynecol 2008; 112(01):201-207

12 Colgan R, Williams M. Diagnosis and treatment of acute uncomplicated cystitis. Am Fam Physician 2011;84(07):771-776

13 American College of Obstetricians and Gynecologists. ACOG Practice Bulletin No. 91: treatment of urinary tract infections in nonpregnant women. Obstet Gynecol 2008;111(03):785-794

14 Vazquez JC, Abalos E. Treatments for symptomatic urinary tract infections during pregnancy. Cochrane Database Syst Rev 2011; 2011(01):CD002256

15 Hill JB, Sheffield JS, McIntire DD, Wendel GD Jr. Acute pyelonephritis in pregnancy. Obstet Gynecol 2005;105(01):18-23 